\title{
Peuple et ethos collectif dans la rhétorique de l'action collective : l'exemple du mouvement de l'été 2011 en Israël
}

\section{Eithan Orkibi}

\section{(2) OpenEdition \\ Journals}

Édition électronique

URL : http://journals.openedition.org/rhetorique/469

DOI : $10.4000 /$ rhetorique.469

ISSN : 2270-6909

Éditeur

UGA Éditions/Université Grenoble Alpes

Édition imprimée

ISBN : 978-2-37747-084-6

Référence électronique

Eithan Orkibi, « Peuple et ethos collectif dans la rhétorique de l'action collective : l'exemple du mouvement de l'été 2011 en Israël », Exercices de rhétorique [En ligne], 7 | 2016, mis en ligne le 26 mai 2016, consulté le 12 septembre 2020. URL : http://journals.openedition.org/rhetorique/469; DOI : https://doi.org/10.4000/rhetorique.469

Ce document a été généré automatiquement le 12 septembre 2020.

\section{c) (†) (9)}

Les contenus de la revue Exercices de rhétorique sont mis à disposition selon les termes de la Licence Creative Commons Attribution - Pas d'Utilisation Commerciale - Partage dans les Mêmes Conditions 4.0 International. 


\title{
Peuple et ethos collectif dans la rhétorique de l'action collective : l'exemple du mouvement de l'été 2011 en Israël
}

\author{
Eithan Orkibi
}

\section{Introduction}

1 Le mouvement social qui s'est développé en Israël durant l'été 2011 a été l'une des plus grandes et des plus longues mobilisations qu'a connues la société israélienne. Partiellement influencé par l'agitation globale - du printemps arabe jusqu'aux mobilisations des «indignés » en Europe -, il a donné lieu pour la première fois à des rassemblements de masse récurrents dans les rues de plusieurs villes israéliennes, pour une série de manifestations et d'actions collectives de grande ampleur autour des questions économiques et sociales.

2 Le mouvement commence le 14 juillet - date iconique qui servira de nom à l'association qui anima une partie des actions, le «J14» - à partir de l'initiative d'une jeune TelAvivienne qui invite ses camarades à dresser des tentes sur le boulevard Rothschild, au cœur d'un quartier huppé du centre de Tel Aviv, pour exprimer la détresse de la jeunesse israélienne face aux prix exorbitants des logements. L'initiative de cette jeune femme, Daphnée Leef, est suivie par des étudiants, militants et adhérents - d'abord à Tel Aviv, mais assez rapidement dans d'autres villes, dans ce qui reçoit le nom de la «révolte des tentes ». Des "villes de tentes » sont installées partout en Israël, dans lesquelles diverses activités sont organisées: meetings et réunions, soirées de conférences et discussions, concerts spontanés et projections de films, et rencontres avec des politiciens et intellectuels. Tous les samedis soirs, à partir de fin juillet et jusqu'à début septembre, des marches ont été organisées pour protester contre le coût de la vie, et exiger des réformes économiques. Sous le slogan «Le peuple demande la 
justice sociale ", des dizaines de milliers de manifestants se sont réunis chaque semaine dans une série de manifestations qui ont atteint leur apogée le 3 septembre, dans un événement de grande envergure baptisé par les médias israéliens «la marche du million ${ }^{1} »$.

Le succès de la mobilisation, somme toute sans précédent dans la réalité israélienne, a sans doute porté quelque peu atteinte à la cohérence idéologique du mouvement. Ce qui aurait pu être une forte revendication de réformes sociales-démocratiques face à l'économie néolibérale dirigée par le gouvernement de Benyamin Netanyahou, s'est transformé en une expérience collective certes novatrice, mais néanmoins limitée à des demandes relativement abstraites sur le plan économique, et soucieuse d'éviter tout appel explicite à un changement politique ${ }^{2}$. Cependant, bien que le mouvement ait réuni les masses autour d'un appel au caractère idéologique ou politique assez fluide, il n'en a pas moins constitué un certain espace de citoyenneté active. Comme l'avait noté l'écrivain israélien Yoram Kaniuk, « les tentes ont constitué la première révolte. Je ne sais pas encore où cela nous mène, mais il s'est passé quelque chose qui nous lie et réunit. [...] C'était la première protestation du peuple israélien ${ }^{3}$ ». À travers les slogans et les discours des leaders du mouvement, et à travers les réactions médiatiques et commentaires des intellectuels, le terme de " peuple » en est venu à occuper une place centrale dans le contexte du mouvement d'été 2011 : en plus de la justice sociale et de réformes économiques, a suggéré l'anthropologue israélien Zali Gurevitch, « le peuple a demandé à être un peuple ${ }^{4}$.

4 C'est à cette figure du peuple, telle qu'elle a été développée et utilisée dans la rhétorique du mouvement d'été 2011, qu'est consacrée la présente étude. On examinera la construction, à travers la parole militante, de la signification du terme de "peuple ", et ses usages argumentatifs. Plus précisément, on s'intéressera ici au rôle que remplit le terme de "peuple» dans la construction stratégique de l'image publique du mouvement. Partant d'une perspective rhétorique, cette étude se situe à la croisée de deux approches théoriques : l'analyse rhétorique des mouvements sociaux et l'analyse $\mathrm{du}$ discours, pour étudier la construction de l'ethos collectif d'un mouvement qui se donne pour objectif d'exprimer la voix du peuple. Centrée sur la figure du "peuple» dans la communication persuasive du mouvement, l'étude utilise la notion d'ethos collectif pour mieux cerner les fonctions qu'a eues «le peuple» dans la formation de l'identité, de l'impression et de l'efficacité persuasive de cette immense protestation.

\section{Cadre théorique}

\subsection{Le discours du peuple dans la rhétorique des mouvements sociaux}

Dans le domaine de la rhétorique des mouvements sociaux ${ }^{5}$, le terme de "peuple » est réservé au discours provenant des milieux non institutionnels et informels qui regroupent des citoyens et des militants autour d'une action collective contre les institutions du Pouvoir. Lorsque les théoriciens et chercheurs en rhétorique aux ÉtatsUnis commencent, dans les années 1950 et 1960, à s'intéresser au discours du quotidien et à la parole de ceux qui ne font pas forcement partie de la catégorie exclusive des "grands hommes", ils se tournent vers le mode de persuasion qui caractérise un contexte jusqu'alors inexploré du point de vue rhétorique: l'action collective. Les 
travaux issus de ce courant de recherche dégagent, depuis les années 1970, la spécificité de la "rhétorique des mouvements sociaux", considérée tout d'abord comme rhétorique collective, dans la mesure où elle représente une collectivité plus ou moins organisée autour d'une cause commune, et dans la mesure où c'est la collectivité qui est souvent reconnue comme la source des messages («les Verts contestent...», «les ouvrières déclarent... », «l'UNEF demande...»). Cette rhétorique est définie comme non-institutionnelle dans la mesure où elle représente une force sociale qui est, de par sa nature, opposée au Pouvoir, mobilisée contre l'ordre établi ou en lutte contre les élites politique, économique et sociale.

6 Ce sont ces deux caractéristiques - sa nature collective et non-institutionnelle - qui font de la rhétorique des mouvements sociaux un espace privilégié pour l'étude de la rhétorique dite "du peuple». Ainsi, les études de la rhétorique des mouvements sociaux se focalisent sur trois dimensions considérées comme spécifiques du discours $\mathrm{du}$ peuple: sa nature conflictuelle, son style "populaire», et son raisonnement "vernaculaire» :

- Souvent traité de « rhétorique coercitive $e^{6} »$ ou " rhétorique de confrontation ${ }^{7} »$, le caractère conflictuel du discours militant, celui qui cherche à influencer par la force en éveillant l'esprit combatif des militants, se manifeste à travers des tactiques langagières de caractère agressif, comme l'appel à la force, les ultimatums, l'incitation à la désobéissance ou à la violence, aussi bien que par des comportements symboliques visant à faire pression sur les institutions comme les marches, l'occupation des locaux, le blocage des rues ou les sit-in, dont l'objectif est d'influencer à travers la perturbation de l'espace urbain8 .

- La deuxième dimension est liée au style non-formel du discours protestataire, et plus particulièrement à l'usage des insultes et des mots obscènes. Le caractère " populaire » de la rhétorique protestataire réside alors dans sa capacité à violer de manière agressive certains tabous sociaux et adopter un comportement discursif visiblement antithétique de celui qu'instaurent les institutions et le pouvoir : «l'obscénité exprime alors un profond mépris pour les normes de la société, une révolte contre l'autorité, et une irrévérence pour les choses sacrées ", précise Dan Rothwell dans son étude consacrée à l'obscénité dans le discours protestataire ${ }^{9}$. Instrument permettant aux militants de transformer leur espace discursif en un lieu d'opposition aux normes sociales, les obscénités, les insultes et les grossièretés, traits caractéristiques d'un sociolecte «bas » ou « informel ${ }^{10}$ », peuvent servir aussi d'instrument stratégique permettant à un mouvement de se présenter, dans l'espace public, comme l'expression authentique d'un sentiment populaire ${ }^{11}$.

- La troisième dimension est liée à ce qu'il est convenu d'appeler, sous l'influence des travaux de Gerard A. Hauser, la rhétorique vernaculaire des mouvements sociaux. Il s'agit d'étudier la manière dont les mouvements sociaux suscitent, véhiculent et reflètent la formation de l'opinion publique dans le discours de ceux qui «n'ont pas de statut officiel», et dont la communication dialogique, relativement spontanée, reflète des "processus collectifs de raisonnement ${ }^{12}$ ». L'un des apports les plus importants de cette approche est sa focalisation sur des acteurs sociaux jusqu'alors moins étudiés dans les recherches portant sur les discours protestataires, à savoir: les adhérents et les militants de base ${ }^{13}$, et les modalités selon lesquelles ils expriment et élaborent ensemble des critiques et revendications sociales. Deux caractéristiques de la rhétorique vernaculaire sont importantes pour la présente étude : d'abord, la liberté prise par rapport aux discours officiels et institutionnels, ce qui permet d'adopter différentes formes discursives comme l'humour et la parodie qui détournent, pour leur résister, les processus « officiels » de raisonnement. Deuxièmement, la polyphonie (polyvocality), c'est-à-dire la multiplicité de voix - positions, opinions, 
revendications - qui caractérisent un espace dynamique, libéré des hiérarchies de la structure sociale, où chaque participant est doté de sa propre "intention auctoriale" (authorial intention ${ }^{14}$ ).

7 Comme on le constate, dans la rhétorique des mouvements sociaux, le terme de " peuple » est associé avant tout à la nature non-formelle et anti-institutionnelle qui caractérise le discours de l'action collective comme cas spécifique de «discours du peuple ». Il s'agit, en l'occurrence, du rejet des processus de prises de décisions et canaux de communication institutionnelle propres aux démocraties libérales, en faveur de modalités de communication "non-traditionnelles» et "moins rationnelles » qui permettent aux groupes marginalisés d'influencer ou de faire pression sur les institutions par l'usage de la rhétorique coercitive et de la violence symbolique. Il s'agit aussi du rejet des règles du discours "civilisé » et du refus du " décorum discursif ", dominés tous deux par la culture officielle, à travers un style moins formel qui reflète l'esprit d'une contre-culture et entend exprimer la voix du peuple. Il s'agit, enfin, d'une rhétorique vernaculaire, polyphonique, qui conteste la structure sociale et le processus de raisonnement institutionnel en créant un espace libre, dynamique et accessible à tous.

\subsection{Du « discours du peuple » à l'« image du peuple »: la question de l'ethos collectif}

Or, bien que ces caractéristiques renvoient à la nature populaire d'un mouvement social, la question ne s'en pose pas moins de savoir si, et jusqu'à quel point, les mouvements de protestation s'engagent dans la construction de leur image publique comme représentants légitimes et reconnus du peuple. En d'autres termes, est-il possible que des traits caractéristiques préalablement perçus comme distinctifs du peuple - style vernaculaire, usage de mots obscènes ou polyphonie -, soient mobilisés non seulement comme formes de communication opposées à celle qu'utilisent traditionnellement les institutions, mais plutôt comme marques d'identité permettant aux mouvements d'apparaître, sur la sphère publique, comme l'expression authentique du peuple? Sur le plan théorique, la présente étude se pose donc la question de savoir quand et comment un mouvement de protestation se donne comme objectif de construire une impression crédible de force sociale populaire.

9 Pour répondre à cette question, nous utilisons la notion d'ethos rhétorique, et plus concrètement, celle d'ethos collectif ${ }^{15}$. Notre point de départ postule que dans la sphère publique, des messages rhétoriques sont produits et diffusés par plusieurs types de sources: des individus (leaders, experts, autorités) aussi bien que des groupes (parti politique, communauté professionnelle, catégorie sociale). Ainsi, si on accepte la logique selon laquelle une image de soi positive ou crédible garantit ou améliore l'efficacité du discours d'un orateur, ce qu'on dénomme "ethos rhétorique ", on peut se demander, à l'instar de Brian Patrick : «If an individual can possess or be possessed by some kind of ethos, then why not a group ${ }^{16}$ ?».

En réalité, la question de l'image de soi des groupes militants est désormais posée dans la rhétorique des mouvements sociaux. Sans forcément évoquer le terme d'ethos, les chercheurs ont développé une série d'outils méthodologiques pour étudier la constitution rhétorique à plusieurs voix de l'identité collective dans un mouvement, et la projection $\mathrm{du}$ "nous» (shared meaning of we-ness ou «signification partagée du 
"nous" ») dans le discours de l'action collective ${ }^{17}$. Néanmoins, bien qu'on examine la construction rhétorique de l'identité en explorant, par exemple, les modalités rhétoriques selon lesquelles les groupes marginaux apprennent à prendre conscience de leurs droits et de leur force politique ${ }^{18}$, on se penche moins sur le rôle de ce «nous » dans la formulation des revendications sociales ou politiques. Autrement dit, c'est l'identité per se qui intéresse les chercheurs plutôt que l'image de soi en tant que moyen de persuasion donnant à un mouvement la légitimité et la crédibilité de faire entendre la voix du peuple.

11 Ainsi, sur le plan théorique, notre étude adopte une définition assez classique de l'ethos comme produit discursif stratégiquement construit par l'orateur pour faire adhérer l'auditoire à la thèse qu'il défend, mais sur le plan méthodologique, nous adoptons l'approche centrée sur l'analyse du discours qui voit dans l'ethos un phénomène discursif plus large, une image de soi que le locuteur - y compris un locuteur collectif construit dans son discours, tel que défini par Ruth Amossy:

Délibérément ou non, le locuteur effectue ainsi dans son discours une présentation de soi. Que l'image induite des façons de dire facilite, parfois même conditionne la bonne réalisation d'un projet, c'est ce que nul ne peut ignorer qu'à ses dépens. [...] La présentation de soi ne se limite pas pour autant à une technique apprise, à un artifice : elle s'effectue, souvent à l'insu des partenaires, dans les échanges verbaux les plus quotidiens et les plus personnels ${ }^{19}$.

Cette distinction est pertinente dans notre cas, puisqu'elle correspond à la distinction qu'établit Dominique Maingueneau ${ }^{20}$ entre l'ethos dit, qui renvoie à ce que l'orateur donne à savoir de lui-même (traits caractéristiques explicitement assertés dans le discours), et l'ethos montré (ce que l'orateur donne à voir de lui-même à travers sa manière de s'exprimer et son comportement discursif $\left.{ }^{21}\right)$. Cette distinction entre le dit et le montré permet de mieux cerner, dans le cas de l'ethos d'un groupe tel qu'un mouvement social, la différence entre l'image du mouvement telle qu'elle est développée, retravaillée et assertée de manière programmatique, par exemple dans les discours publics des porte-paroles du mouvement, et l'image qui découle de manière plus spontanée et moins réfléchie de la parole et du comportement des militants qui, dans un contexte de mobilisation sociale, ne sont pas forcément soumis à une discipline communicationnelle officielle.

\section{Analyse}

La démarche analytique de notre étude consiste à étudier la construction de l'ethos collectif du mouvement de l'été 2011. Nous partons de l'hypothèse que cet ethos est construit en fonction de préoccupations communicationnelles imposées aux acteurs, et, plus précisément, en réponse à des exigences rhétoriques très concrètes sous la forme de critiques des adversaires ciblant la crédibilité du mouvement et la légitimité de ses leaders. Nous postulons que c'est en réaction à ces contraintes, explorées ci-dessus, que les dirigeants du mouvement commencent à travailler leur propre image, et puis l'image du mouvement tout entier. C'est ainsi qu'une grande partie de l'étude est consacrée à l'analyse du discours des leaders ${ }^{22}$, dont notamment les discours publics lors de manifestions et les interviews dans les médias, qui composent le corpus principal de la recherche. Nous y examinons trois aspects liés à la construction de l' ethos dit du mouvement : le rôle du " peuple » dans la consolidation de la légitimité des leaders; la définition du terme de "peuple» dans la formation de l'image de la 
protestation comme un mouvement authentiquement populaire; et le discours de polarisation qui cherche à délimiter le "nous » face aux « autres ». Une seconde partie de l'étude examine l'image issue de l'action collective sur le terrain. Nous nous focalisons, dans cette partie, sur le dispositif discursif associé plus particulièrement au mouvement de l'été 2011, à savoir les slogans créés et scandés par les militants et manifestants de base.

\subsection{Le souci d'apparaître « authentique » comme contrainte rhétorique} départ, un certain problème d'image. Dans une séance de travail du parti au pouvoir, il a été dit qu' «il n'y a pas de protestation réelle... tout le monde est au Boulevard Rothschild avec son Narguilé et Sushi et dans l'aéroport on ne peut pas trouver de chariot à bagage ${ }^{23}$ "; dans une réunion publique du parti, un député s'est exprimé contre les manifestants en affirmant qu'« on a mobilisé les habitants des kibboutz, avec leurs chemises bleues et bandes rouges, c'est une manifestation politique ${ }^{24} »$. Un article d'opinion publié dans un site d'actualité a qualifié la protestation de « Woodstock 2011 entièrement financé par les organisations de gauche ", dont les participants sont

des jeunes aux cheveux longs, marijuana en main, équipés de Smartphones avec les meilleures applications... il s'agit d'un mouvement dirigé par les organisations de gauche dans le but de renverser le Gouvernement... une telle protestation est tout sauf naïve ${ }^{25}$.

quelques exemples illustrent bien les réserves émises par un certain courant dans l'opinion israélienne par rapport aux intentions et objectifs du mouvement : il n'y a pas de véritable détresse économique, les animateurs du mouvement font partie de la jeunesse bourgeoise et de la gauche tel-avivienne, la lutte dite sociale est désormais politique et destinée à déstabiliser le gouvernement de droite. C'est donc la crédibilité même de la manifestation comme mouvement authentique représentant les Israéliens moyens qui a été mise en question.

Cependant, ces critiques ne sont pas la règle. Pour la plupart, les représentants du parti au pouvoir et les membres du gouvernement ont insisté sur la nature " authentique " du mouvement en affirmant, tout d'abord, que «la détresse des logements est désormais réelle. Il ne s'agit pas d'une cause artificielle que quelqu'un a fabriquée. C'est un vrai problème reconnu par tous ", comme l'a déclaré le Premier Ministre, Benyamin Netanyahou ${ }^{26}$. Par rapport aux manifestants, il a été dès le départ affirmé que «c'est une protestation réelle, authentique, menée par la jeunesse qui pense qu'il est temps de faire un changement... il faut mettre le citoyen au centre ${ }^{27} »$. De telles affirmations ont été posées de manière systématique durant tout l'été par les leaders politiques, les membres du gouvernement et les chefs des services publics, faisant ainsi écho aux réactions très favorables que la protestation a suscitées dans les médias. En fait, selon un sondage mené par la chaîne de télévision 10, une majorité presque absolue de la population israélienne ( $88 \%$ des électeurs du Likud) était en faveur de la lutte sociale, et une majorité importante $(70 \%)$ considérait le mouvement comme "véritablement populaire » et comme non « politiquement motivé28 ».

Nous n'avons pas l'objectif de juger ici si ces affirmations représentaient l'avis sincère du gouvernement par rapport au mouvement, ou étaient stratégiquement formulées en 
fonction de la politique communicationnelle du Pouvoir. Nous n'avons pas non plus l'ambition de juger jusqu'à quel point les médias reflétaient ou plutôt formulaient la façon dont la protestation a été perçue par le grand public. Une chose est néanmoins incontestable: la nature « authentique » et le caractère "populaire » du mouvement ont été placés au centre du débat public. Que ce soit en réaction face aux critiques, par une stratégie de marketing social ou par le reflet spontané de son véritable profil, le mouvement lui-même a tout fait pour donner l'impression d'une action sociale du "peuple", d'une lutte dont les leaders comme les manifestants, les porte-paroles comme les sympathisants, représentent l'Israélien moyen par excellence.

Ce souci d'apparaitre " authentique » est devenu l'une des contraintes rhétoriques les plus notables imposées aux leaders du mouvement. Réfutant l'accusation de " politisation ", et visant à mobiliser les secteurs et catégories sociales les plus divers, les leaders se sont efforcés durant l'été d'attribuer à leur mouvement l'image d'une lutte dont l'origine est le «mainstream» de la société israélienne. Le terme de " peuple ", dont la voix est censée être exprimée par la protestation, est devenu le motclé du mouvement. Ce terme, qui habituellement signifie, dans le discours israélien, soit une catégorie ethnique ou nationale (peuple juif ou peuple d'Israël), soit une représentation imaginaire des "gens simples", souvent travailleurs manuels et peu éduqués de la classe inférieure, a changé d'orientation durant l'été, comme le notent les sociologues israéliens Uri Ram et Dani Filk (2013) : pour la première fois, il signifie un sujet démocratique opposé aux élites économique et politique, sans aucune référence à l'ethnos ou volk ${ }^{29}$. Ainsi, le discours du mouvement ne s'est pas seulement référé au terme de "peuple» dans la construction de son image publique, il a également retravaillé la signification de ce terme et le champ herméneutique qui lui est associé.

\subsection{Le « peuple » comme source de légitimité des leaders}

L'une des premières difficultés rencontrées par les leaders du mouvement a été le souci de justifier leur légitimité comme porteurs de revendications sociales issues d'une grande partie de la population israélienne. Le fait que les leaders du mouvement, surtout ceux qui l'avaient initié, faisaient partie du milieu plutôt «bobo » (bourgeoisbohémiens) - comme les définissent Ram et Filk ${ }^{30}$ - jeunes Tel-Aviviens, célibataires, étudiants, journalistes, cinéastes ou professionnels des nouveaux médias, n'a été ignoré ni par les opposants idéologiques, ni par les associations militantes et forces sociales déjà établies. "Ceux qui luttaient pendant des années en faveur de la périphérie sociale ", a-t-on souvent contesté,

sont les vrais militants d'Israël, mais à la télé on ne voit que Daphnée Leef, qui a initié l'action à Rothschild. Pendant des années ils crient et personne ne les écoute. Soudain arrivent les intellos de Tel Aviv et ils prétendent changer le monde ${ }^{31}$.

Traités ainsi de membres de la «bulle tel-avivienne ", l'image stéréotypée des TelAviviens comme hédonistes, égoïstes, individualistes et détachés de la réalité sociale du pays $^{32}$, les leaders du mouvement devaient justifier leur «mandat de revendiquer » et expliquer au nom de qui ils parlent :

Et les deux cents mille personnes qui ont défilé avec nous samedi soir partout dans le pays ? [...] Notre mandat nous a été donné à pied, à travers la mise en place des tentes partout dans le pays. [...] Nous représentons la protestation dans sa totalité, et essayons de le faire de manière transparente et en collaboration avec le public. 
[Par rapport à l'accusation que les médias exagèrent l'ampleur de la protestation] Les médias, c'est aussi des personnes, comme les flics, et nous avons tous les mêmes problèmes. Le fait que les médias nous encouragent, juste comme les flics qui nous disent " bonne chance » après qu'ils nous évacuent de la route, c'est précisément à cause du fait que tous partagent la même détresse populaire ${ }^{33}$.

21 Les leaders ont ainsi justifié leur «mandat " par deux méthodes: d'abord, utiliser l'expansion géographique de la lutte et le nombre grandissant de manifestants, comme preuve de leur qualité et légitimité de leaders. Mais c'est l'autre méthode qui est ici pertinente pour nous: contester les distinctions entre "médias ", "flics » et «militants ", auxquels la question du journaliste ("Vous êtes baptisés 'leaders' surtout par les médias ») présuppose des rôles et objectifs distincts, sinon antagonistes. La réponse de Shaffir qualifie le soutien des médias de solidarité citoyenne, et évoque aussi la sympathie off-record des policiers comme attestation de la capacité du mouvement à rallier différents secteurs et catégories sociales autour d'une cause commune.

La nature «non-sectorielle » des revendications, comme l'a affirmé Shaffir à l'issue de la deuxième semaine du mouvement, deviendra rapidement l'un des éléments constitutifs de son ethos dit et sera assertée à plusieurs reprises. Dans un discours prononcé devant 20000 manifestants dans la ville d'Afoula au Nord du pays - et imprimé le lendemain dans le supplément économique du journal Haaretz, le président de l'Union nationale des étudiants d'Israël, Itzik Shmuli, s'est adressé à «tous les Israéliens ", et a contesté encore plus radicalement les distinctions sociales :

Bonsoir au soldat d'Afoula, bonsoir à l'étudiante de Jérusalem, bonsoir à ses parents du Kibbutz de Dgania, bonsoir au policier de Be'er Sheva, bonsoir à la professeure de Tayibe, bonsoir à l'immigrant d'Ariel, bonsoir au retraité de Netanya, bonsoir au clochard de Holon, bonsoir Israël ! [...] De nombreuses communautés se joignent à nous chaque jour. La détresse commune brise une par une les hautes murailles qui ont divisé la société israélienne. Plus jamais juifs contre arabes, plus jamais laïques contre religieux, plus jamais gauche contre droite. Le coût de la vie nous touche tous et met en évidence la distinction dangereuse entre ceux qui vivent et ceux qui luttent pour vivre dans ce pays ${ }^{34}$.

Ce discours inclusif s'adresse, explicitement, aux différents secteurs de la société tout en contestant les divisions sociales les plus profondes. Cette pratique remplit deux fonctions par rapport à la consolidation de la légitimité des leaders. Premièrement, dans la mesure où le mouvement se fait l'image d'une lutte non-sectorielle qui rassemble tous les Israéliens, l'appartenance sociale des leaders devient a fortiori un paramètre négligeable : il est marginal pour l'évaluation de leur légitimité de dirigeants sociaux. Deuxièmement, ils projettent l'image de dirigeants non seulement en prise sur la réalité sociale et capables de communiquer avec les différentes secteurs de la population, mais aussi et surtout motivés par l'ambition de rétablir une solidarité véritable entre des catégories sociales perçues comme aliénées les unes par rapport aux autres.

\subsection{Le récit constitutif du « peuple »}

24 Ce thème de l'« unité à la place de la division » devient progressivement le récit constitutif (constitutive narrative ${ }^{35}$ ) du mouvement tout entier, voire un instrument de mobilisation qui facilite le recrutement des militants et des adhérents à travers une formule narrative qui attribue un rôle actif aux destinataires. Ces derniers apprennent, 
à travers ce récit mobilisateur, que leur participation au mouvement facilite un processus social plus large de solidarisation. Dans un discours prononcé à l'apogée de la protestation, durant l'immense manifestation tenue à Tel Aviv le 3 septembre ( $« \mathrm{La}$ marche du million »), la leader iconique du mouvement, Daphnée Leef, s'est prononcée en ces termes :

Ma génération a été élevée dans le sentiment que nous sommes seuls dans le monde. C'est chacun devant son écran. L'autre est l'ennemi, le concurrent. [...] Il ne faut faire confiance à personne. [...] Le fait que c'est cette génération - la génération la plus isolée et retirée - qui s'est levée pour agir, est miraculeux. C'est le miracle de l'été 2011. [...] Nous étions fermés chacun dans son propre milieu, dans son milieu d'insatisfaction et d'absurde. Soudain, nous avons commencé à parler, et plus important encore : nous avons commencé à écouter.

Ainsi, le récit fondateur du "peuple», tel qu'il est constitué dans le discours du mouvement, consiste en un moment de prise de conscience qui amène à une libération :

Nous sommes emprisonnés dans notre classe sociale, dans notre lieu de résidence, dans notre religion et dans notre genre. J'ai soudain réalisé que nous ne sommes pas simplement enfermés, mais qu'on nous a emprisonnés. [...] Et au moment où nous avons compris cela, au moment où nous avons commencé à penser ensemble sur notre lendemain, nous nous sommes tous libérés ${ }^{36}$ !

Dans le récit du mouvement, la prise de conscience établit un moment de passage, durant lequel les «Israéliens », jusqu'alors soumis à des cadres cognitifs qui leur ont été imposés pendant des années, constatent l'artificialité des divisions sociales établies, contestent les distinctions qui les ont séparés, rétablissent leurs solidarités et retrouvent leur unité en tant que peuple. Ce motif de la «renaissance» de la citoyenneté israélienne deviendra omniprésent dans le discours du mouvement. Chez Daphnée Leef, par exemple, il s'agit de rejeter la perception exprimée dans la formule "le petit citoyen ", qui souligne, en Israël, l'impuissance du citoyen ordinaire face aux institutions bureaucratiques et son incapacité à résister aux pressions sociales, économiques et politiques :

Le citoyen n'est pas petit! C'est lui qui est le plus grand! Être un citoyen, un grand citoyen, c'est notre mission. Cette protestation exprime le fait que nous ne sommes plus prêts à jouer les petits citoyens, consommateurs, secteurs... ${ }^{37}$

\subsection{Polarisation : le " peuple » et ses « autres »}

Cette constitution de "nous le peuple", qui renonce aux divisions et revendique l'unité, n'est pas sans délimiter un " autre » duquel on le dissocie. Comme nous venons de le voir, il s'agit en premier lieu du rejet de la perception précédente du «nous ». Chez Leef, la formule péjorative "le petit citoyen ", associée à la citoyenneté passive, incompétente et désolidarisée, est remplacée par un détournement de la formule : «le grand citoyen " auquel le mouvement a donné naissance. Chez Shmuli, l'opposition entre l'Israélien du passé et celui constitué par le mouvement est encore plus développée. Dans un discours intitulé «les nouveaux Israéliens", diffusé, comme le discours de Leef, en direct à la télévision durant la « marche du million », il s'adresse au Premier Ministre et déclare :

Monsieur le Premier Ministre. Je sais que vous nous regardez en ce moment. Je vous propose de bien nous regarder. Ce que vous voyez maintenant, ce grand public rassemblé ici et partout en Israël, est un public que vous ne connaissez pas du tout.

[...] Monsieur le Premier Ministre - enchanté. Nous sommes les nouveaux Israéliens.

[...] Nous, les nouveaux Israéliens, nous ne céderons plus, même si on essaye de 
nous décourager. Les nouveaux Israéliens demandent un changement, et ils ne vont plus s'arrêter de lutter ${ }^{38}$. le Premier Ministre, en le menaçant de " vous faire sentir comme au Caire... Si vous ne réparez pas ce que vous avez détruit, vous êtes licencié ${ }^{42}$ ! » Le Premier Ministre et son gouvernement ont été visés dès le début du mouvement, et accusés d'être à la source de tous les maux de la société, y compris, par exemple, "la négligence envers les personnes âgées, les malades, les immigrés et les faibles». On leur reproche d'avoir délibérément créé et cultivé la « détresse économique en fonction de l'intérêt du système économique ${ }^{43}$ ». Le Premier Ministre et ses alliés dans l'élite économique du pays, baptisés "les tycoons", sont également accusés d'avoir découragé la protestation, de s'être moqués des manifestants et d'avoir diabolisé les leaders du mouvement, et ceci dans l'objectif de «nous diviser pour nous opprimer ${ }^{44}$ ». Ainsi, c'est Netanyahou et son gouvernement qui ont rempli, dans la rhétorique du mouvement, le rôle de l'ennemi.

31 Or, cette transformation de Netanyahou et son gouvernement en cible contribue non seulement à l'unification des militants autour d'un ennemi commun, mais aussi à la délimitation du peuple représenté par le mouvement. Vers la fin du mouvement, Itzik Shmuli, renforcé par les masses qui se sont rassemblées pour « la marche du million », s'adresse directement au Premier Ministre, sachant que ses propos sont transmis en direct dans les médias, et pose des ultimatums au gouvernement :

Vous allez trouver des solutions aux problèmes, et nous allons rentrer chez nous. Et si vous n'arrivez pas à trouver des solutions, nous allons rester ici! [...] Maintenant, après cette immense manifestation de force, c'est nous qui allons indiquer au gouvernement la destination, et c'est au gouvernement de nous amener à cette destination. Maintenant, c'est au gouvernement d'agir ${ }^{45}$.

Cette demande, voire cet ultimatum, adressée directement au gouvernement, illustre ainsi le rôle du discours polarisateur dans la construction de l'ethos du mouvement : ce 
sont les citoyens contre le gouvernement, les masses contre les institutions du Pouvoir, le peuple contre l'élite politique et économique. C'est ainsi que s'est constituée l'image du peuple comme sujet démocratique, une catégorie à laquelle appartiennent tous ceux qui ne font pas partie du milieu dirigeant du pays. C'est la définition la plus vaste et la plus inclusive du «nous" qu'un mouvement de protestation peut offrir sur le plan identitaire et sur le plan rhétorique. Étant donnée la fonction mobilisatrice du discours militant, il s'agit de la définition la moins exclusive et la moins ségrégative qui existe : aucun secteur ni aucune catégorie sociale ne sont a priori considérés comme " antagonistes" au mouvement, donc aucune affiliation idéologique, religieuse ou culturelle ne pourra empêcher l'adhésion aux revendications et la participation aux activités.

\subsection{La polyphonie comme ethos montré d'un mouvement populaire} mouvement à travers la multiplicité des revendications. L'espace discursif du mouvement, physiquement actif sur les champs de tentes dressées dans le centre de Tel Aviv et partout dans le pays, s'est rapidement transformé en un véritable «marché des problèmes publics ${ }^{46} "$, où des individus, aussi bien que des associations et organisations, ont exprimé leur propres revendications et ajouté leurs propres interprétations à la revendication plus ou moins généralisée qu'exprime le slogan principal du mouvement : «le peuple demande la justice sociale ». C'est en raison de la polyphonie que le slogan principal a été souvent détourné, parodiquement, en « le peuple demande toutes sortes de choses » ou « le peuple demande n'importe quoi ».

tesse des positions, des causes défendues et des idéologies auxquelles le mouvement a donné lieu a été l'un des traits caractéristiques le plus marqué de la protestation : un article d'opinion dans Haaretz a salué la transformation du boulevard le plus prestigieux de Tel Aviv en un "boulevard d'idées ${ }^{47}$ », et un autre l'a qualifié de « version tel-avivienne de Hyde Park » :

...c'est-à-dire un cercle incluant plusieurs participants, qui parlent et qui écoutent, tous partie prenante d'un dialogue à voix multiples. Dans la version Tel Aviv de ce Hyde Park, pour faire le cercle il faut un cercle de chaises, qu'on peut élargir ou rétrécir, selon la taille du groupe, grand ou petit, impliqué dans la discussion. Des chaises et tables en plastique, des poufs "beanbag ", des tapis - l'essentiel est qu'il y ait, en plein milieu de la ville, un lieu dédié qui permette ce nouveau dialogue entre citoyens ${ }^{48}$.

La manifestation la plus notable de cette polyphonie est le recours aux slogans comme dispositif discursif privilégié des militants de base. Collés sur les murs, sur les tentes, affirmés sur les affiches, écrits très souvent à la main et dans un style simple, plusieurs centaines de slogans ont été créés: les individus et les associations ont inventé et constamment renouvelé leurs propres slogans, puis cité et détourné les slogans des autres. 

courant aux références tirées de la culture israélienne. La majorité des slogans renvoient ou détournent des proverbes traditionnels, des éléments liés à la mémoire collective ou à la culture populaire contemporaine. Ces slogans encadrent les différentes revendications à l'intérieur d'un système herméneutique accessible, reconnu et particulièrement associé au folklore israélien :

Si vous voulez - il n'y a pas! [D'après la célèbre phrase de Theodore Herzl, Si vous voulez, ce ne sera pas une légende, qui symbolise l'aspiration à créer un État juif.]

Ré, rév, révo, révolution! [D’après la formule Na Nah Nahma Nahman Meouman, affiliée au mouvement hassidique de Breslav, mais très représentée aussi dans la culture populaire.]

Il fait cher là-bas [D'après le hit pop Il fait froid là-bas, les mots « cher » [yakar] et « froid» $[k a r]$ se ressemblent en hébreu.]

Toi et moi - on changera le gouvernement [D'après une célèbre chanson israélienne intitulée Toi et moi - on changera le monde.]

\section{Conclusions}

\subsection{Ethos du peuple et ethos populaire}

Cette analyse a montré que la figure du peuple a été centrale dans le discours de (et sur) le mouvement de l'été 2011 en Israël. Initialement, la formule « du peuple » a été mobilisée par les leaders du mouvement afin de consolider leur légitimité comme leaders reconnus, ainsi que pour renforcer la crédibilité du mouvement comme expression authentique du peuple. Or, les leaders ne se sont pas seulement servi du terme de "peuple» pour assurer leur autorité discursive et la légitimité du mouvement, ils ont aussi retravaillé la signification de ce terme, en développant un récit mobilisateur au centre duquel figure la protestation comme un moment singulier de prise de conscience et de renaissance de la citoyenneté israélienne. À partir de ce moment, le «peuple» israélien n'est plus fondé sur les critères qui prévalaient auparavant, tels que «ethnos» ou «religion», mais sur l'appartenance à une collectivité démocratique opposée aux élites politique et économique du pays. Cet ethos de « peuple » qui s'unit pour reprendre son destin en main, rejette les divisions sociales 
établies et évite toute polarisation qui risque d'exclure tel ou tel secteur ou catégorie sociale du collectif constitué par le mouvement. Ainsi, la rhétorique de polarisation du mouvement a été organisée d'une manière diachronique ( nouvelle perception de nous " vs "perception précédente de nous »), et strictement limitée à des cibles concrètes, notamment le Premier Ministre et son gouvernement. Alors que cet ethos est plutôt asserté par les leaders, le discours des militants, celui qui découle des slogans, a fait au contraire émerger un ethos montré. La polyphonie, le style vernaculaire et les références culturelles des slogans ont reflété le caractère populaire de la protestation et renforcé son image comme espace discursif animé par le peuple.

41 Les résultats de l'analyse permettent alors d'établir une distinction entre deux figures distinctes du "peuple» dans la rhétorique des mouvements sociaux. La première est liée à la signification de "peuple ", l'entité sociale au nom de laquelle le mouvement parle, telle qu'elle est mobilisée ou retravaillée par le mouvement. Cette figure du peuple est particulière, et renvoie au cadre social, culturel et national au sein duquel la protestation prend place : il s'agit d'un peuple spécifique (israélien, dans notre cas). La seconde figure est plutôt liée à la signification du caractère "populaire » dans une société donnée. Le mouvement se fait l'image d'une collectivité qui reflète de manière authentique la vox populi, à savoir, la voix d'un courant d'opinion situé au sein de la société civile. Sans ignorer des nuances et les particularités qui distinguent une société de l'autre, nous pouvons conclure que cette figure du peuple s'élabore dans ce qu'il est convenu de considérer dans les démocraties contemporaines comme le discours populaire ou vernaculaire: modes d'expression non-institutionnels, registre informel, espace discursif libéré des contraintes imposées par le discours institutionnel.

42 Ainsi, notre première conclusion consiste à distinguer entre "ethos du peuple » et " ethos populaire ». Le premier correspond aux modalités selon lesquelles le mouvement et ses porte-paroles mobilisent ou construisent l'image du " peuple » au nom duquel ils parlent, à savoir, dans notre cas, le "sujet démocratique » qui s'unit et se solidarise contre « les élites ». L'ethos populaire correspond, par contre, à la capacité du discours protestataire de transmettre l'image d'un mouvement non-institutionnel situé au sein de la société civile, exprimant de manière authentique la vox populi.

\subsection{Vox populi et argument ad populum}

Or, comme nous venons de le voir, la construction de ces deux éthè - ethos du peuple et ethos populaire - est largement située dans un contexte extrêmement concret de mobilisation et se fait en fonction des préoccupations communicationnelles des leaders et des militants. L'ethos, somme toute, doit être considéré comme un instrument rhétorique stratégiquement formé en fonction de besoins persuasifs, et non pas comme l'expression spontanée de l'identité collective du mouvement. Notre cas de figure permet d'indiquer trois fonctions de la figure du peuple dans l'ethos collectif d'un mouvement social :

- La première fonction est liée à l'autorité discursive des leaders, qui élaborent la figure du peuple pour renforcer leur propre légitimité. Nous avons constaté que les dirigeants, qualifiés par leurs opposants de «bobos tel-aviviens », ont initialement utilisé la figure du peuple comme source de crédibilité, leur permettant de réfuter les critiques lancées par les adversaires : le fait de pouvoir se présenter comme leaders capables de rassembler en dehors 
de leur propre milieu, et capables de mobiliser le peuple à travers le pays tout entier, sert de preuve à l'authenticité du mouvement et à la légitimité de ses dirigeants.

- La deuxième fonction est liée à l'identification : l'ethos du peuple et l'ethos populaire renvoient à des représentations collectives non seulement accessibles et connues, mais aussi inclusives. Que la figure du peuple soit imaginaire ou empirique, dans la mesure où le mouvement parvient à être perçu comme exprimant l'esprit et les aspirations du peuple dans le sens le plus large du terme - il est également perçu comme moins sectoriel. La fonction mobilisatrice de l'ethos du peuple est particulièrement visible, dans notre cas, à travers le récit constitutif, qui offre aux adhérents un rôle dans une expérience collective historique: la renaissance de la citoyenneté israélienne à travers l'unification du peuple jusqu'alors divisé en secteurs.

- La troisième fonction est liée à la structure argumentative de la revendication sociale: dans la mesure où les revendications sociales sont idéologiquement et politiquement marquées, pouvoir les exprimer au nom du peuple réduit largement leur caractère subjectif. Comment, et à quel titre, est-il possible de réfuter une revendication - en faveur de logements accessibles ou contre la cherté du coût de la vie - qui est associée dans la sphère publique avec le peuple plutôt qu'avec un acteur précis? Le fait que la revendication est perçue comme l'objet d'un consensus social renforce ainsi sa crédibilité en tant qu'argument dans le débat public. La construction de l'ethos du peuple ou ethos populaire permet d'organiser les revendications du mouvement sous la forme d'arguments éthotiques de type ad populum ${ }^{49}$ : les prémisses sont vérifiées ( la détresse est partagée et admise par tous »), et la conclusion est validée (« donc, les réformes sont nécessaires »).

Ainsi, en conclusion, nous constatons que la figure du peuple dans la rhétorique des mouvements sociaux est l'un des éléments constitutifs de l'ethos collectif du mouvement. Le mouvement et ses dirigeants l'élaborent pour assurer leurs crédibilité et légitimité, pour mobiliser les adhérents et manifestants, et pour présenter leurs messages comme l'expression de la volonté du peuple. Les modalités par lesquelles les mouvements mobilisent et retravaillent, rejettent et renouvellent les figures du peuple en fonction de leurs propres objectifs rhétoriques méritent d'être approfondies. Mais il est également pertinent de souligner, à l'issue de cette analyse, ce que nous rappellent Ron Eyerman et Andrew Jamison : au-delà du contexte immédiat de l'organisation, des objectifs persuasifs et des fonctions rhétoriques, les mouvements constituent des " espaces publics provisoires, des moments de création collective qui fournissent aux sociétés des idées, des identités et même des idéaux ${ }^{50}$ \%. De même que la figure du peuple sert d'instrument rhétorique stratégiquement utilisé par les animateurs de l'action collective, cette figure, une fois créée par le mouvement, participe pleinement à la construction collective de l'imaginaire social.

\section{NOTES}

1. Sur l'émergence, le déroulement et la dispersion du mouvement, on lira E. Y. Alimi, «'Occupy Israel' : A Tale of Startling Success and Hopeful Failure ", Social Movement Studies, $\mathrm{n}^{\circ}$ 11, 3-4, 2012, p. 402-407. 
2. Voir, en particulier, U. Gordon, «Israel's 'Tent Protest' : The Chilling Effect of Nationalism », Social Movement Studies, $\mathrm{n}^{\circ}$ 11, 3-4, 2012, p. 349-55.

3. E. Nehoshtai, « Deux pays pour deux peuples : pourquoi tout le monde déteste Tel Aviv? », The Marker, 12 novembre 2011, http://www.themarker.com/markerweek/1.1562922, nous soulignons.

4. Ibid.

5. Sur l'évolution et les enjeux du domaine, voir R. cox \& C. R. Foust, "Social Movement Rhetoric ", dans A. A. Lunsford, K. H. Wilson \& R. A. Eberly éd., The SAGE Handbook of Rhetorical Studies, Los Angeles, Sage Publications, 2009, p. 605-622.

6. P. G. Burgess, «Crisis Rhetoric: Coercion vs Force », Quarterly Journal of Speech, $\mathrm{n}^{\circ}$ 59, 1, 1973, p. 61-73.

7. R. S. Cathcart, "Movements: Confrontation as Rhetorical Form", Southern Speech Communication Journal, $\mathrm{n}^{\circ} 43,3,1978$, p. 233-247.

8. H. A. Bosmajian éd., Dissent: Symbolic Behavior and Rhetorical Strategies, Boston, Allyn and Bacon, 1971 ; pour une discussion sur la légitimité et la justification éthique de ces tactiques protestataires d'un point de vue rhétorique, voir F. S. Haiman, «'The Rhetoric of the Streets' : Some Legal and Ethical Considerations ", Quarterly Journal of Speech, $n^{\circ}$ 53, 2, 1967, p. 99-114.

9. J. D. Rothwell, «Verbal Obscenity: Time for Second thoughts », Western Speech, n 35, 4, 1971, p. 233.

10. J. Culpeper, D. Bousfield \& A. Wichmann, « "Impoliteness Revisited : With Special Reference to Dynamic and Prosodic Aspects ", Journal of Pragmatics, n³5, 10-11, 2003, p. 1546.

11. E. Orkibi, «Violence verbale et mouvements sociaux: une approche rhétorique », dans B. Fracchiolla, C. Moïse, C. Schultz-Romain \& N. Auger éd., Violences verbales: analyses, enjeux et perspectives, Rennes, Presses Universitaires de Rennes, 2013, p. 55-68.

12. G. A. Hauser, «Vernacular Dialogue and the Rhetoricality of Public Opinion », Communication Monographs, $\mathrm{n}^{\circ}$ 65, 2, 1998, p. 86.

13. Voir, notamment, Herbert W. Simons, « Requirements, Problems, and Strategies : A theory of persuasion for social movements », Quarterly Journal of Speech, n 56, 1, 1971, p. 1-11.

14. G. A. Hauser \& E. D. McClellan, «Vernacular Rhetoric and Social Movements : Performance of Resistance in the Rhetoric of the Everyday ", dans S. McKenzie Stevens \& P. Malesh éd., Active Voices: Composing a Rhetoric of Social Movements, New York, SUNY Press, 2009, p. 30.

15. La notion d'ethos collectif a été développée par R. Amossy, La Présentation de Soi. Ethos et identité verbale, Paris, PUF, 2010, ch. 6. Pour l'étude de la rhétorique protestataire, la notion a été utilisée par E. Orkibi, «Ethos collectif et Rhétorique de polarisation : le discours des étudiants en France pendant la guerre d'Algérie ", Argumentation et Analyse du Discours, n 1, 2008 [en ligne : http://aad.revues.org/438], et E. Orkibi, Les étudiants de France et la guerre d'Algérie. Identité et expression collective de l'UNEF (1954-1962), Paris, Syllepse, 2012.

16. «Si un individu peut endosser ou être revêtu de telle ou telle forme d'ethos, alors pourquoi pas un groupe ?»: B. A. Patrick, "Group Ethos and the Communication of Social Action », Small Group Research, $\mathrm{n}^{\circ}$ 37, 5, 2006, p. 425.

17. C. J. Stewart, C. A. Smith \& R. E. Denton, Persuasion and Social Movement, $4^{\mathrm{e}}$ éd., Long Grove (IL), Waveland, 2001, p. 151-161.

18. R. B. Gregg, "The Ego-Function in the Rhetoric of Protest ", Philosophy and Rhetoric, $\mathrm{n}^{\circ} 4,2$, 1971, p. 71-91.

19. R. Amossy, «La notion d'ethos de la rhétorique à l'analyse du discours », dans R. Amossy éd., Images de soi dans le discours. La construction de l'ethos, Lausanne, Delachaux et Niestlé, 1999, p. 9-10.

20. D. Maingueneau, « Problème d'ethos », Pratiques, n 113-114, 2002, p. 55-67.

21. D. Maingueneau, Le Contexte de l'œuvre littéraire. Énonciation, écrivain, société, Paris, Dunod, 1993, p. 138. 
22. Nous focalisons ici sur les discours et les interviews de Daphnée Leef, qui a initié le mouvement, de Stav Shaffir, jeune militante et journaliste, devenue députée de la Knesset en 2013, et Itizk Shmuli, au moment du mouvement président de l'Union nationale des étudiants d'Israël, élu à la Knesset aussi en 2013.

23. A. Somfalvi, «A Turnmoil in Likud», Ynet, $1^{\mathrm{er}}$ août 2011, http://www.ynet.co.il/articles/ $0,7340, \mathrm{~L}-4103048,00 . \mathrm{html}$. Nous traduisons, ici comme pour toutes les citations qui suivent.

24. A. Somfalvi, « Likud Insists : The Demonstrators - Leftits and Kibbutz Members », Ynet, 4 août 2011, http://www.ynet.co.il/articles/0,7340,L-4104750,00.html.

25. A. Taub, « The Protest of Spoiled Leftists », Nana-10, 27 juillet 2011, http://news.nana10.co.il/ Article/?ArticleID=817777.

26. Conférence de presse, le 26 juillet 2011, diffusée en direct sur la chaîne 2, vers $18 \mathrm{~h}$.

27. Sivan Shalom, Vice-Premier ministre, dans l'émission Meet the Press, le 31 juillet 2011.

28. «Polls : 88\% of Likud Voters support the Tent Protest», Ynet, 2 août 2011, http:// www.ynet.co.il/articles/0,7340,L-4103617,00.html.

29. U. Ram \& D. Filk, "The 14th of July of Daphni Leef: The Rise and the Fall of the Social Protest », Theory \& Criticism, n 41, 2013, p. 20 (en hébreu).

30. Ibid., p. 20-21.

31. T. Heruti-Sover, "They Stole Their Revolution", The Marker, 8 août 2011, http:// www.themarker.com/news/1.678573 (nous soulignons).

32. Pour une analyse de l'opinion publique vis-à-vis du mouvement et de l'image de ses leaders, on lira C. Haber, E. Heller \& T. Herman, «Solidarity in the 2011 Protest : Between Imagination and Reality», The Israel Democracy Institute, 17 décembre 2011, http://www.idi.org.il/media/ 295941.pdf.

33. Extraits d'interview de Stav Shaffir, I. Lior, «Stav Shaffir, Who Authorized You to Demand Anything from the Government?», Haaretz, 2 août 2011, http://www.haaretz.co.il/news/ education/1.1371203.

34. I. Shmuli, «Le discours d'Afoula ", The Marker, 13 août 2011, http://www.themarker.com/ news/protest/1.682536.

35. Le récit constitutif est celui dont les héros et les événements reflètent les valeurs et les aspirations sur lesquelles une communauté est fondée. Voir B. Schwartz, "Social Change and Collective Memory: The Democratization of George Washington », American Sociological Review, vol. 56, 2, 1991, p. 222.

36. D. Leef, « Nous avons trouvé la maison », Ynet, 4 septembre 2011, http://www.ynet.co.il/ articles/0,7340,L-4117387,00.html.

37. Ibid.

38. Cité par T. Litman, «Les nouveaux Israéliens: le discours d'Itzik Shmuli », Calcalist, 3 septembre 2011, http://www.calcalist.co.il/local/articles/0,7340,L-3530257,00.html.

39. C. J. Stewart, C. A. Smith \& R. E. Denton, Persuasion and Social Movement, op. cit., p. 161-167.

40. M. L. Vanderford, "Vilification and social movements : A case study of pro-life and prochoice rhetoric ", Quarterly Journal of Speech, $\mathrm{n}^{\circ}$ 75, 2, 1989, p. 166-182.

41. J. W. Bowers, D. J. Ochs \& R. J. Jensen, The Rhetoric of Agitation and Control, $2^{\mathrm{e}}$ éd., Long Grove (IL), Waveland, 1971, p. 34.

42. D. Leef, Discours du 23 juillet 2011, disponible sur https://www.youtube.com/watch? $\mathrm{v}=\mathrm{HNzmKcAqakg}$.

43. D. Leef, « Nous avons trouvé la maison », op. cit.

44. Ibid.

45. I. Shmuli, cité par T. Litman, « Les nouveaux Israéliens », op. cit.

46. "Social Problems Market» est la métaphore utilisée dans la sociologie des problèmes publics pour désigner la structure compétitive du travail de revendication sociale sur la sphère publique. 
Voir : S. Hilgartner \& C. L. Bost, «The Rise and Fall of Social Problems : A Public Arenas Model », American Journal of Sociology, $\mathrm{n}^{\circ}$ 94, 1, 1988, p. 53-78.

47. T. Shadmi, «Le boulevard d'idées », Haaretz, 16 septembre 2011, http://www.haaretz.co.il/ opinions/1.1475268.

48. "... a circle of many participants, speakers and listeners interested in a multisided dialogue. In the Tel Aviv version, you need a circle of chairs, which can be expanded or contracted for large discussions or small groups. Plastic furniture, beanbag chairs, rugs - what is important is that there is a set place for the people in the middle of the city to conduct the new civic dialogue »: F. Oz-Salzberger, "A New Israeli Voice Has Risen, and Must Not Die ", Haaretz, 20 septembre 2011, http://www.haaretz.com/printedition/opinion/a-new-israeli-voice-has-risen-and-must-not-die-1.385448.

49. À l'instar de Douglas Walton, nous identifions l'argument ad populum comme type d'appel à l'opinion populaire ( $\mathrm{A}$ is true/false because everybody accepts or rejects A »), voir : D. Walton, Appeal to Popular Opinion, University Park, Pennsylvania State University Press, 1999, p. 224.

50. R. Eyerman \& A. Jamision, Social Movements: A Cognitive Approach, University Park, Pennsylvania State University Press, 1991, p. 4.

\section{AUTEUR}

\section{EITHAN ORKIBI}

Université d'Ariel 